\title{
Variables psicosociales y rendimiento académico asociados al optimismo en estudiantes universitarios españoles de nuevo ingreso
}

\author{
MANUEL RAFAEL DE BESA GUTIÉRREZ \\ Universidad de Cádiz, España \\ ORCID: https://orcid.org/0000-0003-1552-2664 \\ JAVIER GIL FLORES \\ Universidad de Sevilla, España \\ ORCID: https://orcid.org/0000-0003-0755-4367 \\ ALFONSO JAVIER GARCÍA GONZÁLEZ* \\ Universidad de Sevilla, España \\ ORCID: https://orcid.org/0000-0002-0839-162X
}

\begin{abstract}
How to quote this article: De Besa Gutiérrez, M.R., Gil Flores, J. \& García González, A.J. (2019). Variables psicosociales y rendimiento académico asociados al optimismo en estudiantes universitarios españoles de nuevo ingreso. Acta Colombiana de Psicología, 22(1), 152-163. doi: http://www.dx.doi.org/10.14718/ACP.2019.22.1.8
\end{abstract}

Resumen

El alumnado de nuevo ingreso encuentra dificultades al inicio de sus estudios universitarios debido a que tiene que afrontar situaciones que generan un alto nivel de estrés, así como nuevos desafíos académicos y sociales o expectativas no satisfechas. Todos estos obstáculos pueden repercutir en su adaptación al contexto universitario y en un bajo rendimiento académico, e incluso llevar al abandono prematuro por parte del estudiante. Según la literatura sobre el tema, entre las variables de carácter psicosocial que intervienen en la adaptación y contribuirían a superar la situación estresante se encuentra el optimismo disposicional; y es teniendo esto en cuenta que en el presente estudio se plantea como objetivo analizar las variables que contribuyen a explicar el optimismo con el que inicia los estudios el alumnado universitario de nuevo ingreso. Con este fin, mediante procedimientos de encuesta se recogieron datos sobre variables académicas y psicosociales en una muestra de 750 estudiantes de diferentes titulaciones de la Universidad de Sevilla, al inicio del primer curso en sus respectivas titulaciones. A partir de los datos obtenidos se realizó el cálculo de estadísticos descriptivos básicos y se utilizaron técnicas como el análisis factorial para explorar la dimensionalidad del constructo optimismo o como la regresión logística binaria para identificar variables relevantes en la diferenciación entre estudiantes optimistas y pesimistas. Los resultados muestran que las variables de rendimiento académico previo, apoyo social percibido, autoestima y autoeficacia percibida se asocian al optimismo de los estudiantes. A partir de estos resultados se derivan recomendaciones de cara a la intervención orientadora sobre el alumnado universitario de nuevo ingreso para favorecer su adaptación al contexto universitario.

Palabras clave: optimismo, variables psicosociales, rendimiento académico previo, educación superior, estudiantes universitarios.

\section{Psychosocial variables and academic performance related to optimism in Spanish first-year university students}

\begin{abstract}
First-year students find difficulties at the beginning of their university studies, facing situations that generate a high level of stress, new academic and social challenges, or unfulfilled expectations. Among the psychosocial variables that intervene in the adaptation and contribute to overcoming the situation is dispositional optimism. The aim of this study was to analyze which variables contribute to explaining the optimism with which first-year university students start their studies. Through survey procedures data were collected on academic and psychosocial variables for a sample of 750 students of different degrees from the University of Seville at the time they started the first course in their respective degrees. The calculation of basic descriptive statistics was used, along with techniques such as factor analysis, to explore the dimensionality of the optimism construct, and binary logistic regression to identify relevant variables in the differentiation between optimistic and pessimistic students. The results show how previous academic performance, perceived social support, self-esteem and perceived self-efficacy are associated with the students' optimism. Based on these results, recommendations are derived for the orientation intervention concerning first-year university students to favor their adaptation to the university context. Keywords: optimism, psychosocial factors, pre-university achievement, higher education, university students.
\end{abstract}

\footnotetext{
* Facultad Ciencias de la Educación, C/ Pirotecnia s/n 41013, Sevilla, alfonsoj@us.es
} 


\title{
Variáveis psicossociais e desempenho acadêmico associados ao otimismo em estudantes universitários espanhóis de novo ingresso
}

Resumo

\begin{abstract}
O alunado de novo ingresso encontra dificuldades no início de seus estudos universitários, pois tem que enfrentar situações que geram alto nível de stress bem como novos desafios acadêmicos e sociais ou expectativas não satisfeitas. Todos esses obstáculos podem repercutir em sua adaptação ao contexto universitário e num baixo desempenho acadêmico, inclusive levar ao abandono precoce do curso. Segundo a literatura sobre o tema, entre as variáveis de caráter psicossocial que intervêm na adaptação e que contribuiriam para superar a situação estressante, encontra-se o otimismo disposicional; nesse sentido, este estudo propõe como objetivo analisar as variáveis que contribuem para explicar o otimismo com o qual o alunado universitário de novo ingresso começa seus estudos. Com esse objetivo, mediante procedimentos de pesquisa, foram coletados dados sobre variáveis acadêmicas e psicossociais numa amostra de 750 estudantes de diferentes cursos. A partir disso, foi realizado o cálculo de estatísticos descritivos básicos e foram utilizadas técnicas como a análise fatorial para explorar a dimensionalidade do constructo otimismo ou como a regressão logística binária para identificar variáveis relevantes na diferenciação entre estudantes otimistas e pessimistas. Os resultados mostram que as variáveis de desempenho acadêmico prévio, apoio social percebido, autoestima e autoeficácia percebida são associados ao otimismo dos estudantes. Com base nesses resultados, foram elaboradas recomendações quanto à intervenção orientadora sobre o alunado universitário de novo ingresso para favorecer sua adaptação ao contexto universitário.

Palavras-chave: otimismo, variáveis psicossociais, desempenho acadêmico prévio, ensino superior, estudantes universitários.
\end{abstract}

\section{Introducción}

El acceso a la educación superior supone para el estudiante de nuevo ingreso un momento de incertidumbre en el que debe afrontar los retos y las nuevas situaciones que implica cursar una titulación universitaria en un entorno que es hasta entonces desconocido. Johnston (2013) hace referencia a los diferentes tipos de cambios que vive el estudiante en su transición a la universidad, entre los que se encuentran los cambios académicos, sociales y personales. En este nuevo escenario, el alumnado que comienza su primer año de estudios en la universidad debe superar algunas dificultades para lograr alcanzar sus metas académicas con éxito (Gairín, Muñoz, Feixas \& Guillamón, 2009), como los altos niveles de estrés que podría llegar a experimentar ante la elevada demanda de actividad que generan los nuevos desafíos (García-Ros, Pérez-González, Pérez-Blasco \& Natividad, 2012), o la insatisfacción y frustración debidas a que no siempre se van a cumplir las altas expectativas académicas iniciales con las que llega el estudiante (Alfonso et al., 2013). En conjunto, estos problemas pueden llevar a una pobre adaptación o incluso al abandono académico de los estudiantes de primer año (Buote et al., 2007), quienes son, de hecho, el colectivo con la tasa de abandono más alta en el contexto europeo (Comisión Europea/EACEA/ Eurydice, 2015).

Esta situación ha llevado a que se plantee el estudio de los factores que contribuyen a la reducción de la deserción universitaria, por lo que se han encontrado estudios recientes en la literatura que han identificado relaciones entre la motivación académica, la procrastinación, los estilos de afrontamiento (Montgomery et al., 2017), la satisfacción con la vida, el significado de la vida (Bailey \& Phillips, 2016), las habilidades en mindfulness (Ramler, Tennison, Lynch \& Murphy, 2016), el engagement y la inteligencia emocional (Perera \& DiGiacomo, 2015) con la adaptación del alumnado al entorno universitario. Específicamente, la revisión llevada a cabo por Credé y Niehorster (2012) agrupa en ocho categorías las diferentes variables -utilizadas en estudios previos- relacionadas con el ajuste universitario, dentro de las cuales destaca un grupo que trata sobre los rasgos de personalidad y las autoevaluaciones de los propios estudiantes, sobre todo en lo que respecta a la autoestima, la autoeficacia y el optimismo.

En este sentido, el optimismo disposicional se ha identificado como una variable relevante tanto para un mejor ajuste del estudiante al entorno universitario, como para su rendimiento académico (Londoño, 2009). En particular, Carver y Scheier (2014) definen el optimismo disposicional como un constructo cognitivo que engloba las expectativas con respecto a resultados futuros; definición complementada por Londoño (2009), quien añade que estas expectativas de buenos resultados futuros se dan incluso ante situaciones y circunstancias de adversidad.

De este modo, a la hora de actuar, las personas optimistas atribuyen las causas negativas de un acontecimiento a 
154

orígenes externos, mientras que los sujetos poco optimistas tienden a explicarlas culpándose a sí mismos (Seligman, 2003). Laranjeira (2008), además, destaca los beneficios del optimismo sobre la salud, ya que ha encontrado relaciones entre este constructo y el bienestar tanto psicológico como físico y social de las personas evaluadas.

En el contexto de la educación superior, investigaciones anteriores han aportado evidencias acerca de los beneficios del optimismo disposicional para el alumnado universitario, ya que, por ejemplo, han constatado que este constructo contribuye a la prevención del abandono académico (Londoño, 2009; Roso-Bas, Jiménez \& García-Buades, 2016), a mejorar la adaptación dentro del contexto universitario (Chemers, Hu \& García, 2001; Montgomery, Haemmerlie \& Ray, 2003; Perera \& McIlveen, 2014), e, incluso, a aumentar el rendimiento académico de los estudiantes (Guillén, PérezLuzardo \& Arnaiz, 2013; Monteiro, Tavares \& Pereira, 2008; Solberg, Evans \& Segerstrom, 2009).

En esta línea, Brissette, Scheier y Carver (2002) resaltan el poder amortiguador que ejerce el optimismo ante situaciones de estrés, y señalan que el optimismo ayuda a manejar nuevos entornos, como el universitario, al aumentar el uso de estrategias activas de afrontamiento y reducir los niveles de estrés.

\section{Optimismo y contexto universitario}

La literatura científica ha identificado variables relevantes por su relación con el optimismo dentro del contexto universitario. En lo que respecta a los rasgos demográficos de los estudiantes, se han explorado las diferencias en el optimismo en función del género, pero los resultados no apuntan de manera consistente en una u otra dirección, ya que, por ejemplo, mientras los resultados del estudio de Puskar et al. (2010) evidencian menos niveles de optimismo en mujeres que en hombres, otros trabajos no encuentran diferencias significativas (Huan, Yeo, Ang \& Chong, 2006; Patton, Bartrum \& Creed, 2004). Por otra parte, en cuanto a la edad, el estudio de Londoño (2009) sobre alumnado universitario mostró una correlación positiva entre la edad y el optimismo, al igual que los resultados del estudio de Zou et al. (2016), donde se muestra una relación lineal positiva del optimismo con esta variable; lo que quiere decir que a medida que aumenta la edad del sujeto, el grado de optimismo también es mayor. Estos resultados respaldan empíricamente los planteamientos de autores como Seligman (2003) y Martin (2002), donde se explica el optimismo como una característica aprendida en la edad adulta.

También, se ha analizado la relación entre el optimismo y otras variables psicosociales en los estudiantes, y, específicamente, se ha encontrado que el optimismo, en conexión con altos niveles de apoyo social, incrementa el bienestar subjetivo de estudiantes universitarios (Marrero \& Carballeira, 2010) y permite afrontar con éxito las demandas caracterizadas como estresantes (FernándezGonzález, González-Hernández \& Trianes-Torres, 2015). En este sentido, el planteamiento de Chapman y Chi (2017) muestra relevancia al explicar cómo el apoyo social percibido actúa como mecanismo para que las personas optimistas afronten de manera más activa los desafíos que trae consigo el día a día.

De igual forma, las expectativas optimistas de futuro en los estudiantes universitarios se han asociado a una alta autoestima (Montgomery et al., 2003), ya que se han evidenciado dichas relaciones de forma moderada en diferentes trabajos empíricos tanto en el contexto universitario (Kapikiran \& Acun-Kapikiran, 2016; Liu et al., 2017) como en estudiantes de educación secundaria (Monzani, Steca \& Greco, 2014); y otra variable que ha centrado la atención de los investigadores es la autoeficacia percibida, ya que, por ejemplo, analizando la adaptación al contexto universitario en el alumnado de nuevo ingreso, Morton, Mergler y Boman (2014) constataron la relación directa entre autoeficacia y optimismo, además de una relación entre ambas variables y la adaptación universitaria; Jovanović y Gavrilov-Jerković (2013) hallaron correlaciones positivas entre la autoeficacia percibida y el optimismo disposicional en una muestra compuesta por estudiantes universitarios y de secundaria; y Pu, Hou y Ma (2017), igualmente, respaldaron la relación entre la autoeficacia y el optimismo disposicional, aunque argumentaron que es la autoeficacia la que promueve una visión optimista, y que, de modo inverso, una baja autoeficacia explicaría una perspectiva pesimista del individuo.

Asimismo, la conexión entre el optimismo y los logros académicos es otro ámbito de estudio que ha centrado el interés de los investigadores; $y$ en este caso, Monteiro et al. (2008) encontraron una correlación positiva y significativa entre ambas variables, concluyendo que aquellos estudiantes que presentaban niveles altos de optimismo mostraban un mayor rendimiento académico al finalizar su semestre académico; al igual que Chemers et al. (2001), quienes encontraron que el optimismo en conjunto con la autoeficacia resultaron ser factores tenidos en cuenta para explicar la adaptación universitaria y el rendimiento académico en el alumnado de nuevo ingreso, ya que los resultados señalaron que ambas variables inciden en el rendimiento académico $\mathrm{y}$ en el ajuste de los estudiantes al nuevo contexto.

Además de esto, y de forma consistente, la literatura ha señalado el poder del optimismo como variable que favorece la adaptación universitaria del estudiante de nuevo ingreso, a la vez que diferentes autores que han realizado trabajos empíricos en el área derivan de sus resultados la 
recomendación de diseñar e implementar programas que promuevan el optimismo (Fernández-González et al., 2015; Monteiro et al., 2008; Perera \& McIlveen, 2014).

En este sentido, resulta de gran interés identificar los factores que se asocian en mayor medida al optimismo con el fin de concretar algunos de los aspectos que le den contenido a programas o estrategias de intervención en este ámbito, y es por esto que en el presente trabajo se planteó como objetivo analizar la relevancia de diferentes variables psicosociales y académicas en la explicación del optimismo con el que el alumnado afronta el inicio de sus estudios universitarios.

\section{Método}

\section{Diseño}

Se realizó un estudio de corte cuantitativo y ex-post facto, con un diseño de tipo descriptivo-correlacional, según la tipología recogida por Hernández, Fernández y Baptista (2003), y de carácter transversal, dado que la recogida de datos se llevó a cabo en un único momento.

\section{Participantes}

La población considerada en este estudio se ubica en el contexto español y está constituida por el alumnado que accede por primera vez a una titulación universitaria de Grado en la Universidad de Sevilla. Por medio de un procedimiento de muestreo no probabilístico, y atendiendo a los criterios de accesibilidad, se constituyó una muestra de 750 estudiantes universitarios de nuevo ingreso de las cinco áreas de enseñanza universitaria (véase Tabla 1). No se incluyó en la muestra a los estudiantes de primer curso que fueran repitentes, que tuvieran otra titulación universitaria ya terminada ni que provinieran de otras titulaciones de grado no completadas. El $57.5 \%$ eran mujeres y el $42.5 \%$, hombres. La media de edad para los participantes es de 18.94 años $(D T=2.20)$.

De acuerdo con los procedimientos de análisis que se describen en un apartado posterior, en la muestra inicial se identificaron dos submuestras de alumnado caracterizadas por un alto optimismo (247 estudiantes) o un bajo optimismo (248 estudiantes).

\section{Instrumentos}

Se consideró como variable dependiente el optimismo disposicional, el cual se midió por medio del Test de Orientación Vital (LOT-R), en su versión revisada (Scheier, Carver \& Bridges, 1994). El test está formado por 10 ítems con una escala de respuesta tipo Likert, de los cuales tres se refieren al optimismo, tres al pesimismo, y cuatro sirven para hacer menos evidente el contenido del test y no son considerados a efectos de puntuación. Las respuestas oscilan entre muy en desacuerdo (1) y muy de acuerdo (5). Para este instrumento, Scheier et al. (1994) obtuvieron una fiabilidad estimada por alfa de Cronbach de .78., y en el presente trabajo se obtuvo un valor de alfa de .75 para la muestra total. Este valor puede considerarse aceptable, teniendo en cuenta que la medida obtenida con el test LOT-R se apoya únicamente en seis ítems.

Scheier et al. (1994) plantearon una estructura unifactorial, entendiendo que el constructo optimismo-pesimismo constituye un solo rasgo con dos polos; sin embargo, la adaptación al castellano empleada en el presente estudio es la utilizada por Ferrando, Chico y Tous (2002), y en

Tabla 1.

Muestra de estudiantes universitarios de nuevo ingreso

\begin{tabular}{ccc}
\hline Áreas de conocimiento & Titulaciones & Frecuencia \\
\hline \multirow{2}{*}{ Artes y humanidades } & Estudios ingleses & 25 \\
& Historia del arte & 34 \\
Ciencias sociales y Jurídicas & Derecho & 75 \\
& Educación primaria & 62 \\
Ciencias & Administración y dirección de empresas & 97 \\
& Biología & 25 \\
Ciencias de la salud & Matemáticas & 47 \\
& Química & 50 \\
Ingenierías & Enfermería & 93 \\
& Psicología & 73 \\
\end{tabular}


156

esta, en contra de la estructura unidimensional atribuida originalmente al LOT-R, los citados autores recogen diferentes estudios que plantean la bidimensionalidad del instrumento y constatan la existencia de dos factores que se corresponden con optimismo y pesimismo. Con el objetivo de clarificar este aspecto, en el presente estudio se realizó un análisis factorial exploratorio (AFE) para identificar el número de dimensiones subyacentes a la escala, seguido de un análisis factorial confirmatorio (AFC).

En particular, se seleccionó de manera aleatoria una parte de la muestra-que correspondía al $50 \%$-para realizar el AFE, y con la parte restante se llevó a cabo el AFC; la idoneidad de la matriz de correlaciones para llevar a cabo este análisis se confirmó por medio del test de esfericidad de Barlett $(\chi 2=502.49 ; \mathrm{p}=.000)$ y la medida de adecuación de muestreo de Kaiser-Meyer-Olkin $(\mathrm{KMO}=.80)$. Siguiendo las recomendaciones de Lloret-Segura, FerreresTraver, Hernández-Baeza y Tomás-Marco (2014), para la extracción de factores se utilizó el método de máxima verosimilitud, previa comprobación de que los valores de asimetría (comprendidos en valores absolutos entre .09 y $.78)$ y curtosis (entre .09 y .90 ) permitieran confiar en la normalidad de las variables.

En la solución factorial se retuvo una sola dimensión, teniendo en cuenta que la distancia entre el autovalor del primer factor (2.757) y del segundo (.957) era considerablemente mayor que la registrada entre este y los autovalores para los factores sucesivos (.770 en el tercero y .566 en el cuarto). La retención de un solo factor fue, además, un resultado compatible con el criterio teórico de partida, y los seis ítems presentaron saturaciones altas en el mismo (véase Tabla 2).

Tabla 2.

Saturaciones de los items en la dimensión optimismo

\begin{tabular}{cc}
\hline Ítems & Pesos \\
\hline Siempre soy optimista en cuanto al futuro & .646 \\
En general, espero que me ocurran más cosas buenas & .680 \\
Casi nunca cuento con que me sucedan cosas buenas & .631 \\
En tiempos difíciles, suelo esperar lo mejor & .598 \\
Rara vez espero que las cosas salgan a mi manera & .593 \\
$\begin{array}{c}\text { Si algo malo me tiene que pasar, estoy seguro de que } \\
\text { me pasará }\end{array}$ & .388 \\
\hline
\end{tabular}

El AFC corroboró que el modelo de un solo factor se ajustaba satisfactoriamente a los datos y confirmó la estructura unidimensional de la escala. Los índices de bondad de ajuste mostraron valores aceptables $(\chi 2=53.263$, $\mathrm{p}<.000, \chi 2 / \mathrm{gl}=9$, GFI $=.954, \mathrm{CFI}=.916)$ o muy próxi$\operatorname{mos}(\mathrm{RMR}=.063)$ a los valores que permitirían afirmar la bondad del modelo (Byrne, 2001). En consecuencia, en los análisis del presente estudio se utilizó el rasgo optimismo disposicional como una sola puntuación.

Por otra parte, como variables independientes se incluyeron la autoestima, la autoeficacia, el apoyo social percibido, el rendimiento académico previo y los rasgos demográficos de sexo y edad.

Específicamente, la autoestima se midió por medio de la Escala de Autoestima de Rosenberg (Rosenberg, 1965), en su versión adaptada al español por Martín-Albo, Núñez, Navarro y Grijalvo (2007). Estos autores realizaron dos administraciones del instrumento a una muestra de alumnado universitario y confirmaron su estructura unidimensional al obtener valores de fiabilidad medida con un alfa de Cronbach de .85 y .88 en cada una de ellas. Para el presente estudio, el instrumento arrojó un valor de alfa de .87 . También, es importante mencionar que este instrumento consta de 10 ítems, donde cinco están enunciados de forma positiva y cinco de forma negativa, $y$ que las respuestas de los sujetos se expresan a través de una escala Likert de cuatro puntos que va desde totalmente en desacuerdo (1) hasta totalmente de acuerdo (4).

Adicionalmente, para medir la variable autoeficacia se recurrió a la Escala de Autoeficacia General (Baessler \& Schwarcer, 1996), un instrumento que mide un único constructo a través de 10 ítems que adoptan el formato de una escala tipo Likert con cuatro grados de acuerdo que van desde totalmente en desacuerdo (1) hasta totalmente de acuerdo (4). La adaptación española de este instrumento, utilizada en el presente estudio, es la propuesta por Sanjuán, Pérez y Bermúdez (2000); autores que obtuvieron para el mismo una fiabilidad $\alpha=.87$, similar al valor encontrado en el presente estudio $(\alpha=.84)$.

Y, por último, para la medición de la variable apoyo social percibido se utilizó la Escala de Apoyo Social Familiar y de Amigos (AFA-R), de González-Ramírez y Landero-Hernández (2014), compuesta por 14 ítems referidos al apoyo que reciben de la familia o de los amigos. Los ítems de esta escala se responden utilizando una escala de frecuencia con cinco niveles, donde 1 se corresponde con nunca y 5 con siempre. Al aplicarla con estudiantes universitarios, sus autores hallaron una fiabilidad de .92 para el instrumento, y en el presente estudio, en semejanza con los resultados de consistencia interna, también se encontró un valor de alfa alto $(\alpha=.89)$.

Las variables edad, sexo y rendimiento académico previo se obtuvieron a partir de preguntas formuladas a los participantes encuestados en el momento de cumplimentar los instrumentos de medida; y en el caso del rendimiento académico se les pidió que consignaran la calificación obtenida en la prueba de acceso a la universidad -con la cual accedieron a la titulación de grado que cursaban-. 


\section{Procedimiento}

La recogida de datos se realizó en el segundo cuatrimestre del curso académico 2016-2017, concretamente, durante el mes de abril de 2017. Inicialmente, se contactó mediante correo electrónico al profesorado que se encontraba impartiendo asignaturas de primer año universitario en diferentes titulaciones; $y$, tras su aprobación, se llevó a cabo la aplicación de los cuestionarios dentro del aula, con una duración aproximada de 15 minutos, incluyendo la presentación del estudio, la explicación sobre los instrumentos de recogida de datos y la cumplimentación de los mismos por parte de los estudiantes.

\section{Consideraciones éticas}

De acuerdo con las normas contenidas en el Código de Buenas Prácticas en Investigación de la Universidad de Sevilla (2017), se garantizó el anonimato de los participantes, quienes respondieron a los cuestionarios de manera voluntaria tras haber recibido información sobre el propósito y la naturaleza del estudio. De la participación en el estudio no se derivaron beneficios académicos para los estudiantes, y se garantizó que quienes declinaran participar en el estudio no sufrieran consecuencias adversas por ello. La recogida de datos fue llevada a cabo por los propios investigadores.

\section{Análisis de datos}

Con respecto al análisis, las respuestas a los ítems utilizados en las escalas de autoestima y autoeficacia fueron codificadas de 1 a 4, mientras que las cinco modalidades de respuesta para los ítems relativos al apoyo social percibido y al optimismo fueron codificadas de 1 a 5 , invirtiendo estos valores en el caso de ítems de sentido negativo, de modo que una puntuación alta se corresponde siempre con un nivel alto en el rasgo medido.

Por otra parte, se registró una incidencia de valores perdidos muy baja (inferior al $1 \%$ en todas las variables), por lo que se optó por trabajar con los casos para los que se contaba con información completa en las variables implicadas en cada análisis (procedimiento casewise) como procedimiento general. Así, el análisis inició con el cálculo de estadísticos descriptivos e índices de consistencia interna tanto para la medida de optimismo disposicional como para las otras variables consideradas en el estudio.

La muestra se dividió en tres grupos a partir de las puntuaciones factoriales del optimismo derivadas de un análisis factorial exploratorio similar al aplicado para examinar la unidimensionalidad del instrumento, aunque aplicado esta vez a la totalidad de los participantes. Para ello, se utilizaron los terciles de la distribución, y esto permitió diferenciar al alumnado optimista, al alumnado que no se decanta hacia posiciones optimistas o pesimistas, y al alumnado claramente pesimista. Finalmente, reteniendo a los participantes que se ubicaban en el grupo de alumnado con mayor y menor optimismo, el análisis de regresión logística binaria permitió explorar en qué medida las variables psicosociales y académicas estudiadas contribuyen a la diferenciación entre optimistas y pesimistas. Para el análisis de los datos se utilizaron los programas estadísticos SPSS v.23 y AMOS v.24.

\section{Resultados}

En el siguiente apartado se presentan los resultados del análisis de los datos, comenzando por los estadísticos descriptivos de las variables de estudio, seguidos de las asociaciones entre las distintas variables y el optimismo por medio de un análisis de regresión logística.

\section{Estudio descriptivo de las variables}

Además de las variables demográficas de edad y sexo ya presentadas en la descripción de los participantes en el estudio se recogió información adicional sobre la calificación obtenida en las pruebas de acceso a la universidad. Específicamente, la media de estas puntuaciones alcanza el valor de $10.9(D T=1.69)$ en una escala de puntuación con un máximo de 14.

Por otra parte, en el caso de las variables optimismo, autoestima, autoeficacia y apoyo social, las puntuaciones totales en cada uno de los instrumentos utilizados para su medición fueron divididas por el número de ítems, de manera que quedaron expresadas en valores comprendidos entre 1 y 5 para las variables optimismo y apoyo social, y en valores de 1 a 4 para autoestima y autoeficacia.

La Tabla 3 muestra que los estudiantes universitarios perciben un elevado apoyo de sus familias y amigos, reflejado en una puntuación media de 4.22; tienden a mostrarse optimistas, con una puntuación de 3.39-ubicada ligeramente por encima del valor 3 , que constituye el punto central de la escala-; y se sitúan en niveles elevados en lo que respecta a la autoeficacia y la autoestima -con valores que superan el 3 , en una escala que va de 1 a 4 -.

Tabla 3.

Estadísticos descriptivos

\begin{tabular}{ccc}
\hline Variable & Media & Desviación típica \\
\hline Optimismo disposicional & 3.39 & .72 \\
Autoestima & 3.17 & .54 \\
Autoeficacia & 3.05 & .42 \\
Apoyo social & 4.22 & .66 \\
\hline
\end{tabular}


Explicación del optimismo a partir de las variables psicosociales y académicas

Se recurrió al análisis de regresión logística binaria para valorar la medida en que se asocian al optimismo del alumnado las variables psicosociales y académicas consideradas en este estudio. Específicamente, la variable dependiente binaria fue el optimismo, la cual se diferenció entre el alumnado optimista (valor 1) y el alumnado pesimista (valor 0); y como variables independientes se incluyó el sexo, la edad, el rendimiento académico previo, la autoestima, la autoeficacia y el apoyo social percibido.

De acuerdo con el test de Hosmer y Lemeshow, el modelo construido con estas variables se ajusta adecuadamente a los valores observados, ya que al estadístico de contraste $($ Chi cuadrado $=6.837)$ se asocia un grado de significación $(\mathrm{p}=.554)$ que permite mantener la hipótesis nula de igualdad entre los valores observados y los valores esperados según el modelo. La bondad del modelo se estimó a partir de la $\mathrm{R}$ cuadrado de Cox y Sell $\left(\mathrm{R}^{2}=.384\right)$ y la $\mathrm{R}$ cuadrado de Nagelkerke $\left(\mathrm{R}^{2}=.512\right)$, cuyos valores indican una buena explicación de la variable dependiente por las variables independientes. Además, su potencia explicativa se apoya en el elevado porcentaje de estudiantes correctamente clasificados como optimistas y pesimistas, que se cifran en $81.5 \%$ y $73.3 \%$, respectivamente, con un porcentaje global de pronóstico exitoso para el $77.5 \%$ del alumnado.

En la Tabla 4 se recogen los resultados del análisis de regresión logística, donde se puede observar que las variables con mayor grado de asociación al optimismo disposicional son la autoestima y la autoeficacia $(\mathrm{p}<.001)$. También, resulta significativo el efecto de las variables de apoyo social percibido $(\mathrm{p}=.020) \mathrm{y}$, en menor medida, rendimiento académico previo $(\mathrm{p}=.047)$. En presencia de las restantes variables, los rasgos demográficos de sexo y edad no resultaron relevantes en la explicación del optimismo $(\mathrm{p}>.05)$.
A partir de las odds ratios, es posible cuantificar la importancia de cada una de las variables en la diferenciación de alumnado universitario optimista y pesimista; específicamente, su valor indica qué tan probable es presentar una disposición optimista por cada unidad en que se incremente la puntuación registrada en la variable independiente considerada. Así, el incremento unitario de la puntuación en autoestima supone aproximadamente multiplicar por trece las probabilidades de ser optimista (odds ratio $=13.093$ ), mientras que aumentar un punto en autoeficacia casi quintuplica las probabilidades de que el estudiante se muestre optimista (odds ratio $=4.714$ ). Menos importantes son los efectos del rendimiento previo o el apoyo social (odds ratio de 1.175 y 1.672 , respectivamente).

\section{Discusión}

En general, el alumnado que inicia sus estudios en la Universidad de Sevilla lo hace con una disposición optimista, ya que los niveles de optimismo mostrados por los estudiantes de nuevo ingreso se sitúan por encima del punto medio de la escala utilizada para su medición. Teniendo en cuenta el modo en que se define el constructo optimismo disposicional, este primer resultado lleva a considerar que los nuevos estudiantes acceden a la universidad con expectativas de lograr resultados académicos positivos. Adoptando una perspectiva transcultural, los niveles de optimismo expresados por el alumnado participante en el estudio se aproximan a los constatados en trabajos anteriores llevados a cabo en el contexto anglosajón con estudiantes que cursan su primer año académico en la universidad (Chemers et al., 2001; Solberg et al., 2009; Perera \& McIlveen, 2014), aunque se distancian de los bajos niveles de optimismo hallados para el alumnado de nuevo ingreso en sistemas

Tabla 4.

Coeficientes, odds ratios y $F$ de Wald para los efectos sobre el optimismo

\begin{tabular}{ccccccc}
\hline Variables & \multirow{2}{*}{$\mathrm{B}$} & \multirow{2}{*}{ F de Wald } & \multirow{2}{*}{ Sig. } & \multirow{2}{*}{ Odds ratio } & \multicolumn{2}{c}{ IC 95 \% para odds ratio } \\
\cline { 6 - 7 } & & & & Inferior & Superior \\
\hline Edad $^{1}$ & .488 & 1.782 & 3.255 & 1.629 & .959 & 2.770 \\
Sexo $^{2}$ & .086 & 2.682 & 1.145 & 1.090 & .931 & 1.275 \\
Rendimiento previo $^{1}$ & .161 & 6.941 & 3.951 & 1.175 & 1.002 & 1.378 \\
Autoestima $^{1}$ & 2.572 & 58.746 & 62.385 & 13.093 & 6.916 & 24.787 \\
Autoeficacia $^{1}$ & 1.551 & 16.228 & 18.188 & 4.714 & 2.312 & 9.613 \\
Apoyo social $^{1}$ & .514 & 4.558 & 5.437 & 1.672 & 1.085 & 2.576 \\
Constante & -18.799 & 48.168 & 47.745 & .000 & & \\
\hline
\end{tabular}

Nota. ${ }^{1}$ Odds ratio asociada al incremento de una unidad en la variable independiente $;{ }^{2}$ Categoría de referencia: hombre. 
universitarios latinoamericanos (Gustems-Carnicer, Calderón \& Forn, 2017; Laranjeira, 2008).

Por otra parte, ante la controversia suscitada por la dimensionalidad del factor optimismo disposicional medido a través de la escala LOT-R, los resultados de la presente investigación corroboran la existencia de una sola dimensión bipolar, tal como lo propusieron los autores del instrumento (Scheier et al., 1994).

Ahora bien, partiendo de las medidas obtenidas para el constructo optimismo disposicional, el objetivo fundamental del presente trabajo fue el de establecer las variables que contribuyen a explicar el optimismo, y, de acuerdo con los hallazgos obtenidos, los principales factores vinculados al optimismo expresado por los estudiantes son la autoestima y la autoeficacia. Estos resultados convergen con los aportados en la literatura previa, donde se ha constatado que los estudiantes optimistas presentan mayor autoestima y registran correlaciones significativas de nivel bajo (Mustaca, Kamenetzk \& Vera-Villarroel, 2010) o moderado (Montgomery et al., 2003); y que la autoestima ha venido siendo una variable importante en el estudio de la adaptación al contexto universitario (Friedlander, Reid, Shupak \& Cribbie, 2007; Salami, 2011), por lo que se argumenta que los estudiantes que muestran altos niveles de autoestima se sienten con más confianza y manejan mejor aquellos desafíos que encuentran a su entrada a la educación superior. En este sentido, Hickman, Bartholomae y McKenry (2000) subrayan la importancia de la autoestima en los estudiantes universitarios para conseguir una transición satisfactoria.

En lo que respecta a la autoeficacia percibida, la conexión con el optimismo hallada en este estudio confirma que cuanto más consciente sea el individuo de que posee las habilidades propias para llevar a cabo cualquier acción, más aumentan sus expectativas de resultados. Este resultado refuerza los hallazgos de estudios previos en los que se obtuvieron correlaciones positivas entre autoeficacia y optimismo (Jovanović \& Gavrilov-Jerković, 2013; Morton et al., 2014), en los cuales los estudiantes con altos niveles de autoeficacia observan la entrada a la universidad más como un reto que como una amenaza, y actúan con más confianza y optimismo al manejar los nuevos desafíos y utilizar estrategias más efectivas.

Por otra parte, aunque con un menor poder explicativo, las variables de apoyo social y rendimiento académico previo también resultaron relevantes en relación con el optimismo del alumnado, ya que el hecho de que el estudiante perciba suficiente apoyo social incide en sus niveles de optimismo de manera positiva. Este resultado es compatible con los obtenidos en el estudio de Brissette et al. (2002), quienes muestran que los estudiantes optimistas informan más apoyo social por parte de sus compañeros que aquellos con unas expectativas pesimistas, además de que presentan un mejor ajuste al contexto universitario al desarrollar mayor apoyo social a partir de sus redes de amistades.

También, en la misma línea de trabajos anteriores (Chemers et al., 2001; Monteiro et al., 2008) en los que se halló una relación directa entre el optimismo y el rendimiento académico, los resultados obtenidos muestran, igualmente, la conexión entre ambas variables, ya que el rendimiento académico previo resultó ser un factor con cierta capacidad de explicar el optimismo con el que el alumnado afronta su nueva etapa de estudios universitarios.

A diferencia de lo anterior, con respecto a las variables psicosociales y académicas, los rasgos demográficos como género o edad no resultaron relevantes al diferenciar alumnado optimista y pesimista, y estos resultados se alinean con los antecedentes hallados en la literatura, debido a que señalan una correlación débil (Londoño, 2009; Moreno \& Marrero, 2015) o nula (Huan et al., 2006; Patton et al., 2004; Solberg et al., 2009) entre este tipo de variables demográficas y el optimismo.

Cabe mencionar que el trabajo realizado se realiza desde una perspectiva no habitual en el estudio del optimismo disposicional del alumnado, puesto que en trabajos anteriores se ha utilizado el optimismo generalmente como una variable independiente capaz de predecir aspectos como la adaptación al medio universitario, la permanencia en la universidad, los resultados académicos, y el bienestar personal, social y físico; sin embargo, teniendo en cuenta la importancia del optimismo con respecto al éxito del alumnado de nuevo ingreso, su adaptación al medio universitario y su retención, el presente estudio enfocó la atención sobre las variables que contribuirían a explicar el optimismo y que, por tanto, resultan relevantes a la hora de favorecer el desarrollo de una visión y disposición optimistas en los estudiantes universitarios de nuevo ingreso.

Ahora bien, teniendo en cuenta los resultados obtenidos en el presente estudio, una primera recomendación derivada de los mismos es la necesidad de prestar especial atención al alumnado universitario de nuevo ingreso que cuenta con un pobre rendimiento académico previo, dada la relevancia de esta variable para identificar estudiantes susceptibles de mostrar pesimismo ante la nueva etapa educativa que inician y que, en consecuencia, podría representarles problemas para su integración en el contexto universitario.

Adicionalmente, la literatura explica la existencia de programas que lograron potenciar e incrementar el grado de optimismo en estudiantes universitarios (Remor \& AmorósGómez, 2012; Remor, Amorós-Gómez \& Carrobles, 2010), $y$, en este sentido, otra recomendación de este trabajo alude a los ámbitos en los que se podría intervenir para favorecer el optimismo de los estudiantes de nuevo ingreso, y así potenciar 
el desarrollo de las variables que aquí han demostrado ser relevantes. Por ejemplo, un claro ámbito de intervención tiene que ver con la autoestima que habría de ser fortalecida en el alumnado, por lo que programas de mentoría entre iguales podrían tener incidencia sobre la autoestima tanto del estudiante mentor como del mentorizado, sobre todo para favorecer y fortalecer el desarrollo personal de ambos (Valverde, Ruíz, García \& Romero, 2004).

Por otro lado, ante estudiantes que acceden al contexto universitario con niveles pobres de autoeficacia, RamosSánchez y Nichols (2007) proponen actuaciones basadas en la teoría de Bandura (1986), tales como el aprendizaje vicario o el control de las emociones. Y esto es relevante con respecto al papel de los mentores, ya que se les puede formar para que asuman la enseñanza de técnicas de relajación con el objetivo de reducir la excitación emocional en momentos en que el estudiante perciba como amenazantes. De este modo, compartir conocimientos y habilidades desarrollados durante su experiencia en la universidad podría propiciar un aprendizaje vicario en los estudiantes mentorizados, y, dada la importancia del apoyo social percibido, la intervención desde el contexto universitario habría también de incidir en la mejora del clima relacional entre el alumnado y en el establecimiento de nuevos vínculos de amistad que propicien un apoyo recíproco. En la medida en que este tipo de actuaciones se favorezca el optimismo del alumnado, se contribuiría a mejorar su ajuste al medio universitario, por lo que se favorecería su consecución de logros académicos y se prevendría el abandono prematuro.

Finalmente, el trabajo realizado presenta como fortaleza la amplitud de la muestra utilizada y la heterogeneidad de la misma, aspectos que contribuyen a su representatividad a pesar de no haber sido seleccionada mediante procedimientos aleatorios. Además, se incorporan en un mismo modelo multivariable diferentes factores para estudiar su relación con el optimismo; sin embargo, las principales limitaciones son inherentes a la propia naturaleza del diseño de investigación y de las técnicas de recogida de datos empleadas, ya que, aunque el enfoque correlacional adoptado permite identificar variables que se asocian con el optimismo y que son capaces de explicar su presencia o ausencia en el alumnado universitario, no permite establecer relaciones causa-efecto que proporcionen una base empírica sólida para afirmar la influencia de factores como la autoestima, la autoeficacia o el apoyo social sobre el optimismo. En este sentido, futuros trabajos podrían orientarse hacia el diseño e implementación de intervenciones dirigidas a potenciar los factores mencionados con el fin de valorar por medio de enfoques metodológicos cuasi-experimentales la eficacia de las mismas para favorecer el optimismo del alumnado en el primer año de estudios universitarios. Para terminar, otra línea de trabajo interesante sería la consideración de un mayor número de variables potencialmente asociadas con el optimismo (como el bienestar psicológico, los niveles de estrés, la introversión-extroversión, etc.), para construir modelos de ecuaciones estructurales que den cuenta de las relaciones entre las mismas.

\section{Referencias}

Alfonso, S., Deaño, M., Conde, A., Costa, A. R., Araújo, A. M., \& Almeida, L. S. (2013). Perfiles de expectativas académicas en alumnos españoles y portugueses de enseñanza superior. Revista Galego-portuguesa de Psicoloxía e Educación, 21(1), 125-136. Recuperado de https://ruc.udc.es/dspace/bitstream/handle/2183/12610/RGP_21_2013_art_9. pdf? sequence $=1$ \&isAllowed $=\mathrm{y}$

Baessler, J., \& Schwarcer, R. (1996). Evaluación de la autoeficacia: Adaptación española de la escala de Autoeficacia General. Ansiedad y Estrés, 2, 1-8.

Bailey, T. H., \& Phillips, L. J. (2016). The influence of motivation and adaptation on students' subjective well-being, meaning in life and academic performance. Higher Education Research \& Development, 35(2), 201-216. doi: 10.1080/07294360.2015.1087474

Bandura, A. (1986). Social foundations of thought and action: A social cognitive theory. Englewood Cliffs, NJ: Prentice Hall.

Brissette, I., Scheier, M. F., \& Carver, C. S. (2002). The role of optimism in social network development, coping, and psychological adjustment during a life transition. Journal of Personality and Social Psychology, 82(1), 102-111. doi: 10.1037/0022-3514.82.1.102

Buote, V. M., Pancer, S. M., Pratt, M. W., Adams, G., BirnieLefcovitch, S., Polivy, J., \& Maxine, G. W. (2007). The importance of friends. Journal of Adolescent Research, 22(6), 665-689. doi:10.1177/0743558407306344

Byrne, B. M. (2001). Structural equation modelling with AMOS: Basic concepts, applications, and programming. New York: LEA.

Carver, C. S., \& Scheier, M. F. (2014). Dispositional optimism. Trends in Cognitive Sciences, 18(6), 293-299. doi: 10.1016/j.tics.2014.02.003

Chapman, T., \& Chi, T. C. (2017). Perceived social support mediates the link between optimism and active coping. Journal of Behavioral and Social Sciences, 4, 57-65. Recuperado de https://www.researchgate.net/publication/318090510_Per ceived_Social_Support_Mediates_the_Link_Between_Optimism_and_Active_Coping

Chemers, M. M., Hu, L. T., \& Garcia, B. F. (2001). Academic self-efficacy and first year college student performance and adjustment. Journal of Educational Psychology, 93(1), 5564. doi:10.1037/0022-0663.93.1.55 
Comisión Europea/EACEA/Eurydice (2015). El Espacio Europeo de Educación Superior en 2015: Informe sobre la implantación del Proceso de Bolonia. Luxemburgo: Oficina de Publicaciones de la Unión Europea.

Credé, M., \& Niehorster, S. (2012). Adjustment to college as measured by the student adaptation to college questionnaire: A quantitative review of its structure and relationships with correlates and consequences. Educational Psychology Review, 24(1), 133-165. doi: 10.1007/s10648-011-9184-5

Fernández-González, L., González-Hernández, A., \& TrianesTorres, M. V. (2015). Relaciones entre estrés académico, apoyo social, optimismo-pesimismo y autoestima en estudiantes universitarios. Electronic Journal of Research in Educational Psychology, 13(1), 111-130. doi: 10.14204/ ejrep.35.14053

Ferrando, P. J., Chico, E., \& Tous, J.M. (2002). Propiedades psicométricas del test de optimismo Life Orientation Test (LOT). Psicothema, 14(3), 673-680. Recuperado de http:// www.psicothema.es/pdf/782.pdf

Friedlander, L. J., Reid, G. J., Shupak, N., \& Cribbie, R. (2007). Social support, self-esteem, and stress as predictors of adjustment to university among first-year undergraduates. Journal of College Student Development, 48(3), 259-274. doi: 10.1353/csd.2007.0024

Gairín, J., Muñoz, J. L., Feixas, M., \& Guillamón, C. (2009). La transición secundaria- universidad y la incorporación a la universidad. La acogida de los estudiantes del primer año. Revista Española de Pedagogía, 242, 27-44. Recuperado de https://revistadepedagogia.org/wp-content/ uploads/2009/01/242-03.pdf

García-Ros, R., Pérez-González, J., Pérez-Blasco, F., \& Natividad, L. A. (2012). Evaluación del estrés académico en estudiantes de nueva incorporación a la universidad. Revista Latinoamericana de Psicología, 44(2) 143-154. doi: 10.14349/rlp.v44i2.1038

González-Ramírez, M. T., \& Landero-Hernández, R. (2014). Propiedades psicométricas de la Escala de Apoyo Social Familiar y de Amigos (AFA-R) en una muestra de estudiantes. Acta de Investigación Psicológica, 4(2), 1469-1480. doi: 10.1016/S2007-4719(14)70387-4

Guillén, F., Pérez-Luzardo, J., \& Arnaiz, P. (2013). Relación entre motivación y optimismo disposicional en aprendices de inglés como lengua extranjera en un contexto universitario. Revista de Educación, Número extraordinario, 104-128. doi: 10.4438/1988-592X-RE-2013-EXT-243

Gustems-Carnicer, J., Calderón, C., \& Forn, M. (2017). Psychometric properties of the Life Orientation Test (LOT-R) and its relationship with psychological well-being and academic progress in college students. Revista Latinoamericana de Psicología, 49(1), 19-27. doi: 10.1016/j.rlp.2016.05.001

Hernández, R., Fernández, C., \& Baptista, P. (2003). Metodología de la investigación. México: Mc Graw Hill.

Hickman, G. P., Bartholomae, S., \& McKenry, P. C. (2000). Influence of parenting style on the adjustment and academic achievement of traditional college freshmen. Journal of College Student Development, 41(1), 41-54. Recuperado de http://dropouts101.com/downloads/Influence $\% 20 \mathrm{of} \% 20$ Parenting\%20Styles\%20on\%20Adjustment\%20and\%20 Achievement $\% 20$ of $\% 20$ Traditional $\% 20$ College $\% 20$ Freshmen $\% 20-\% 20$ Journal $\% 20$ of $\% 20$ College $\% 20$ Student $\% 20$ Development.pdf

Huan, V. S., Yeo, L. S., Ang, R. P., \& Chong, W. H. (2006). The influence of dispositional optimism and gender on adolescents 'perception of academic stress. Adolescence, 41(163), 533-546. Recuperado de https://www.researchgate.net/ publication/6576081_The_influence_of_dispositional_optimism_and_gender_on_adolescents\%27_perception_of_ academic_stress

Johnston, B. (2013). El primer año de universidad. Una experiencia positiva de transición. Madrid, España: Narcea.

Jovanović, V., \& Gavrilov-Jerković, V. (2013). Dimensionality and validity of the serbian version of the Life Orientation Test-Revised in a sample of youths. Journal of Happiness Studies, 14(3), 771-782. doi: 10.1007/s10902-012-9354-2

Kapikiran, S., \& Acun-Kapikiran, N. (2016). Optimism and psychological resilience in relation to depressive symptoms in university students: examining the mediating role of selfesteem. Educational Sciences: Theory and Practice, 16(6), 2087-2110. doi: 10.12738/estp.2016.6.0107

Laranjeira, C. A. (2008). Tradução e validação portuguesa do revised Life Orientation Test (LOT-R). Universitas Psychologica, 7(2), 469-476. Recuperado de http://revistas.javeri ana.edu.co/index.php/revPsycho/article/view/469

Liu, Q. Q., Zhou, Z. K., Yang, X. J., Niu, G. F., Tian, Y., \& Fan, C. Y. (2017). Upward social comparison on social network sites and depressive symptoms: A moderated mediation model of self-esteem and optimism. Personality and Individual Differences, 113, 223-228. doi: 10.1016/j. paid.2017.03.037

Londoño, C. (2009). Optimismo y salud positiva como predictores de la adaptación a la vida universitaria. Acta Colombiana de Psicología, 12(1), 95-107. Recuperado de https:// editorial.ucatolica.edu.co/ojsucatolica/revistas_ucatolica/ index.php/acta-colombiana-psicologia/article/view/335

Lloret-Segura, S., Ferreres-Traver, A., Hernández-Baeza, A., \& Tomás-Marco, I. (2014). El Análisis Factorial Exploratorio de los Ítems: una guía práctica, revisada y actualizada. Anales de Psicología, 30(3), 1151-1169. doi: 10.6018/ana lesps.30.3.199361

Marrero, R. J., \& Carballeira, M. (2010). El papel del optimismo y del apoyo social en el bienestar subjetivo. Salud mental, 33(1), 39-46. Recuperado de http://www.inprf-cd.gob. $\mathrm{mx} / \mathrm{pdf} / \mathrm{sm} 3301 / \mathrm{sm} 330139 . \mathrm{pdf}$

Martin, N. (2002). Feeling optimistic? Psychologist. 15(6) 309321.

Martín-Albo, J., Núñez, J., Navarro, J., \& Grijalvo, F. (2007). The Rosenberg Self-Esteem Scale: Translation and validation in 
university students. The Spanish Journal of Psychology, 10(2), 458-467. doi:10.1017/S1138741600006727

Monteiro, S., Tavares, J., \& Pereira, A. (2008). Optimismo disposicional, sintomatologia psicopatológica, bem-estar e rendimento académico em estudantes do primeiro ano do ensino superior. Estudos de Psicologia, 13(1), 23-29. doi:10.1590/S1413-294X2008000100003

Montgomery, R. L., Haemmerlie, F. M., \& Ray, D. M. (2003). Psychological correlates of optimism in college students. Psychological reports, 92(2), 545-547. doi: 10.2466/ pr0.2003.92.2.545

Montgomery, S., Gregg, D. H., Somers, C. L., Pernice-Duca, F., Hoffman, A., \& Beeghly, M. (2017). Intrapersonal Variables Associated with Academic Adjustment in United States College Students. Current Psychology, 1-10. doi: 10.1007/s12144-016-9533-0

Monzani, D., Steca, P., \& Greco, A. (2014). Brief report: assessing dispositional optimism in adolescence - Factor structure and concurrent validity of the Life Orientation Test Revised. Journal of Adolescence, 37, 97-101. doi: 1016/j. adolescence.2013.11.006

Moreno, Y., \& Marrero, R. J. (2015). Optimismo y autoestima como predictores de bienestar personal: diferencias de género. Revista Mexicana de Psicología, 32(1), 27-36. Recuperado de http://www.redalyc.org/pdf/2430/243045363004. pdf

Morton, S., Mergler, A., \& Boman, P. (2014). Managing the transition: The role of optimism and self-efficacy for firstyear Australian university students. Australian Journal of Guidance and Counselling, 24(1), 90-108. doi:.10.1017/ jgc.2013.29

Mustaca, A. E., Kamenetzky, G., \& Vera-Villarroel, P. (2010). Relaciones entre variables positivas y negativas en una muestra de estudiantes argentinos. Revista Argentina de Clínica Psicológica, 19(3), 227-235. Recuperado de http:// www.redalyc.org/articulo.oa?id=281921798004

Patton, W., Bartrum, D. A., \& Creed, P. A. (2004). Gender differences for optimism, self-esteem, expectations and goals in predicting career planning and exploration in adolescents. International Journal for Educational and Vocational Guidance, 4(2), 193-209. doi: 10.1007/s10775-005-1745-z

Perera, H. N., \& DiGiacomo, M. (2015). The role of trait emotional intelligence in academic performance during the university transition: An integrative model of mediation via social support, coping, and adjustment. Personality and Individual Differences, 83, 208-213. doi: 10.1016/j. paid.2015.04.001

Perera, H. N., \& McIlveen, P. (2014). The role of optimism and engagement coping in college adaptation: A career construction model. Journal of Vocational Behavior, 84(3), 395-404. doi: 10.1016/j.jvb.2014.03.002

Pu, J., Hou, H., \& Ma, R. (2017). Direct and Indirect Effects of Self-efficacy on Depression: The Mediating Role of
Dispositional Optimism. Current Psychology, 36(3), 410416. doi: 10.1007/s12144-016-9429-z

Puskar, K. R., Marie Bernardo, L., Ren, D., Haley, T. M., Hetager Tark, K., Switala, J., \& Siemon, L. (2010). Self-esteem and optimism in rural youth: Gender differences. Contemporary Nurse, 34(2), 190-198. doi: 10.5172/conu.2010.34.2.190

Ramler, T. R., Tennison, L. R., Lynch, J., \& Murphy, P. (2016). Mindfulness and the college transition: the efficacy of an adapted mindfulness-based stress reduction intervention in fostering adjustment among first-year students. Mindfulness, 7(1), 179-188. doi: 10.1007/s12671-015-0398-3

Ramos-Sánchez, L., \& Nichols, L. (2007). Self-efficacy of firstgeneration and non-first-generation college students: The relationship with academic performance and college adjustment. Journal of College Counseling, 10(1), 6-18. doi: 10.1002/j.2161-1882. 2007.tb00002.x

Remor, E., \& Amorós-Gómez, M. (2012). Efecto de un programa de intervención para la potenciación de las fortalezas y los recursos psicológicos sobre el estado de ánimo, optimismo, quejas de salud subjetivas y la satisfacción con la vida en estudiantes universitarios. Acta Colombiana de Psicología, 15(2), 75-86. Recuperado de https://editorial. ucatolica.edu.co/ojsucatolica/revistas_ucatolica/index.php/ acta-colombiana-psicologia/article/view/268

Remor, E., Amorós-Gómez, M., \& Carrobles, J.A. (2010). Eficacia de un programa manualizado de intervención en grupo para la potenciación de las fortalezas y recursos psicológicos. Anales de Psicología, 26 (1), 49-57. Recuperado de http://revistas.um.es/analesps/article/view/91961

Rosenberg, M. (1965). Society and the adolescent self-image. Princeton, NJ: Princeton University Press.

Roso-Bas, F., Jiménez, A. P., \& García-Buades, E. (2016). Emotional variables, dropout and academic performance in Spanish nursing students. Nurse Education Today, 37, 5358. doi: 101016/j.nedt.2015.11.021 0260-6917

Salami, S. O. (2011). Psychosocial Predictors of Adjustment among First Year College of Education Students. US-China Education Review, 8(2), 239-248. Recuperado de https:// files.eric.ed.gov/fulltext/ED519567.pdf

Sanjuán P., Pérez A. M., \& Bermúdez J., (2000) Escala de autoeficacia general: datos psicométricos de la adaptación para población española, Psicothema, 12(2), 509-513. Recuperado de http://www.psicothema.com/pdf/615.pdf

Scheier, M. F., Carver, C. S., \& Bridges, M. W. (1994). Distinguishing optimism from neuroticism (and trait anxiety, selfmastery, and self-esteem: a reevaluation of the Life Orientation Test. Journal of Personality and Social Psychology, 67(6), 1063-1078. doi: 10.1037//0022-3514.67.6.1063

Seligman, M.E. (2003). La auténtica felicidad. Barcelona. España: Vergara Editor.

Solberg, L., Evans, D. R., \& Segerstrom, S. C. (2009). Optimism and college retention: Mediation by motivation, performance, and adjustment. Journal of Applied Social 
Psychology, 39(8), 1887-1912. doi: 10.1111/j.15591816.2009.00508.x

Universidad de Sevilla. (2017). Código de buenas prácticas en investigación. España: Vicerrectorado de Investigación, Universidad de Sevilla. Recuperado de https://investigacion. us.es/docs/apoyo/CÓDIGO\%20DE\%20BUENAS\%20 PRÁCTICAS\%20EN\%20INVESTIGACIÓN\%20DE\%20 LA\%20UNIV\%20DE\%20SEVILLA.pdf
Valverde, A., Ruíz, C., García, E., \& Romero, S. (2004). Innovación en la orientación universitaria: La mentoría como respuesta. Contextos educativos, 6(7), 87-112. doi: $10.18172 /$ con. 530

Zou, R., Zhang, D. J., Niu, G. F., Xie, X. C., Fan, C. Y., Tian, Y., \& Zhou, Z. K. (2016). Cross-sectional age differences in dispositional optimism in Chinese children and adolescents. Personality and Individual Differences, 102, 133-138. doi: 10.1016/j.paid.2016.06.063 